\title{
Local infiltration analgesia versus interscalene nerve block for postoperative pain control after shoulder arthroplasty: a prospective, randomized, comparative noninferiority study involving 99 patients
}

\author{
Julia Sicard, MDª, Shahnaz Klouche, MD ${ }^{a, *}$, Christel Conso, MD ${ }^{b}$, Nicolas Billot, MD ${ }^{c}$, \\ Jean-Charles Auregan, MD, PhDa , Samuel Poulain, MD $^{d}$, Florent Lespagnol, MD', \\ Nicolas Solignac, MD $^{c}$, Thomas Bauer, MD, PhD ${ }^{a, f}$, Mathieu Ferrand, MDa , \\ Philippe Hardy, MD, PhD ${ }^{a, f}$
}

\author{
${ }^{a}$ Hôpitaux Universitaires Paris Ile-de-France Ouest, Assistance Publique-Hôpitaux de Paris (AP-HP), \\ Boulogne-Billancourt, France \\ ${ }^{b}$ Institut Mutualiste Montsouris, Paris, France \\ ${ }^{c}$ Clinique Claude Bernard, Ermont, France \\ ${ }^{d}$ Polyclinique du Plateau, Bezons, France \\ ${ }^{e}$ Clinique Jules Verne, Nantes, France \\ ${ }^{f}$ Université de Versailles Saint-Quentin-en-Yvelines, Versailles, France
}

\begin{abstract}
Background: The aim of this study was to compare the efficacy of local infiltration analgesia (LIA) and interscalene nerve block (ISB) for early postoperative pain control after total shoulder arthroplasty (TSA). The hypothesis was that LIA is not inferior to ISB.

Methods: A prospective, randomized controlled study was performed in 2014-2016. All patients who underwent TSA for shoulder osteoarthritis were included. Patients in the ISB group received a continuous infusion of $0.2 \%$ ropivacaine by perineural catheter for 48 hours. The surgeon injected $110 \mathrm{~mL}$ of $0.2 \%$ ropivacaine, $30 \mathrm{mg}$ of ketoprofen, and $0.5 \mathrm{mg}$ of epinephrine before TSA in the LIA group and inserted a catheter into the glenohumeral joint. The next morning, $10 \mathrm{~mL}$ of $0.2 \%$ ropivacaine, $30 \mathrm{mg}$ of ketoprofen, and epinephrine were injected through the catheter, which was then removed. The primary outcome was the mean shoulder pain score for the 48-hour postoperative period on a numerical scale (0-10). The secondary outcomes were postoperative opioid requirements, complications, and shoulder function at the 1-month follow-up visit. The sample size was calculated for a noninferiority study.
\end{abstract}

This study was approved by the institutional review board of Comité de Protection des Personnes Ile de France IV, Hôpital Saint-Louis, Paris, France.
*Reprint requests: Shahnaz Klouche, MD, Hôpitaux Universitaires Paris Ile-de-France Ouest, Assistance Publique-Hôpitaux de Paris (AP-HP), 9 Avenue Charles-de-Gaulle, Boulogne-Billancourt 92100, France.

E-mail address: klouche_shahnaz@yahoo.fr (S. Klouche).

1058-2746/\$ - see front matter @ 2018 Journal of Shoulder and Elbow Surgery Board of Trustees. All rights reserved.

https://doi.org/10.1016/j.jse.2018.09.026 
Results: The study included 99 patients (50 LIA and 49 ISB patients) with a mean age of $72 \pm 9.6$ years. Although no significant difference in the mean pain score was found between the 2 groups for the 48hour postoperative period ( $1.4 \pm 0.9$ for LIA vs $1.7 \pm 1$ for ISB, $P=.19$ ), the LIA group had significantly less severe pain $(P=.003)$ and less opioid consumption $(P=.01)$ in the recovery room. No complications occurred. A negative but nonsignificant correlation was found between postoperative pain and Constant score at the 1-month follow-up.

Conclusion: LIA is not less effective than ISB for early postoperative pain control after TSA.

Level of evidence: Level II; Randomized Controlled Trial; Treatment Study

(C) 2018 Journal of Shoulder and Elbow Surgery Board of Trustees. All rights reserved.

Keywords: Total shoulder arthroplasty; local infiltration analgesia; interscalene nerve block; early postoperative pain; opioid consumption; noninferiority study

Total shoulder arthroplasty (TSA) may result in severe pain. ${ }^{15}$ Interscalene nerve block (ISB) is the reference technique for pain control during TSA.9.11 According to the metaanalysis of Abdallah et al, ${ }^{1}$ rebound pain could appear during the first few hours after the effects of an ISB disappear. Insertion of a perineural catheter makes it possible to prolong analgesia up to 48 hours after surgery. ${ }^{8}$ However, this technique has certain-sometimes permanent and, in particular, neurologic — adverse events due to spreading of the local anesthetic into adjacent nerve structures or as a result of the act of insertion itself. ${ }^{13}$ Sviggum et $\mathrm{al}^{20}$ reported that the overall incidence of perioperative nerve injury after TSA was $2.2 \%$ (95\% confidence interval, 1.6\%-3.1\%) and was unrelated to ISB use.

Injection of local infiltration analgesia (LIA) into the surgical site has become increasingly frequent in the past few years. In situ diffusion of LIA blocks the nociceptive nerve message at the distal nerve ends and does not result in any paresthesia or muscle weakness in the involved limb. Effective pain control has been confirmed with this technique in arthroplasty of the hip and knee, with reduced morphine consumption compared with morphine analgesia 24 hours after surgery, as well as a shorter hospital stay. ${ }^{5,7}$ Pain control can be prolonged by inserting a catheter into the glenohumeral joint and readministering the product the day after surgery. ${ }^{7}$

The goal of this study was to compare the efficacy of LIA and ISB in the control of early postoperative pain after TSA. We hypothesized that LIA was not less effective than ISB for this indication.

\section{Materials and methods}

We performed a prospective, randomized controlled noninferiority study in 4 centers specialized in shoulder surgery. The included patients were all aged over 18 years and underwent shoulder arthroplasty because of osteoarthritis of the shoulder, with either anatomic or reverse arthroplasty in the case of large rotator cuff tears. The exclusion criteria were as follows: contraindication to ISB (respiratory failure or peripheral oxygen saturation $<90 \%$ in ambient air), history of allergy to one of the treatments in the study, chronic opioid treatment for more than 3 months, history of shoulder surgery on the same side, weight of less than $50 \mathrm{~kg}$, American Society of
Anesthesiologists score greater than 3 , and/or incapacity to provide informed consent and pain self-assessment. All patients provided informed consent. The protocol was filed in a register of international clinical studies (https://clinicaltrials.gov, identifier 02365181). CONSORT (Consolidated Standards of Reporting Trials) guidelines were followed.

\section{Anesthesia protocol}

Surgery was performed with a standardized general anesthesia protocol. Induction was achieved with $2-3 \mathrm{mg} / \mathrm{kg}$ of propofol and $0.2-$ $0.3 \mu \mathrm{g} / \mathrm{kg}$ of sufentanil, which could be administered again during surgery at the discretion of the anesthesiologist; $0.5 \mathrm{mg} / \mathrm{kg}$ of atracurium; and $0.15 \mathrm{mg} / \mathrm{kg}$ of ketamine. Then, anesthesia was maintained by inhalation of a halogenated anesthetic to obtain a minimal alveolar concentration of 1-1.5. Patients were given $8 \mathrm{mg}$ of dexamethasone to prevent postoperative nausea and vomiting. Prophylactic antibiotics were administered with a slow intravenous infusion of $2 \mathrm{~g}$ of cefazolin or $900 \mathrm{mg}$ of clindamycin. All patients wore support socks or stockings during the procedure.

\section{Surgical technique}

Surgery was performed by senior surgeons specialized in shoulder arthroplasty. The patient was in the beach-chair position during surgery with the arm on a hydraulic arm holder. A deltopectoral surgical approach was used. A tenotomy of the subscapularis tendon was performed in all cases with systematic repair for anatomic arthroplasties and at the discretion of the surgeon for reverse arthroplasties.

The shoulder was immobilized after the procedure, and physical rehabilitation during external rotation was limited depending on the condition of the subscapularis tendon. Mobilization of the shoulder and physical rehabilitation for everyday activities (dressing, toileting) were begun the day after surgery.

\section{ISB protocol}

A perineural interscalene catheter was inserted before surgery under ultrasound control by a senior anesthesiologist. The anesthesiologist injected $20 \mathrm{~mL}$ of $0.2 \%$ ropivacaine during the TSA procedure after a negative aspiration test to confirm the absence of blood reflux in the catheter. In the recovery room, a continuous perfusion of $0.2 \%$ 

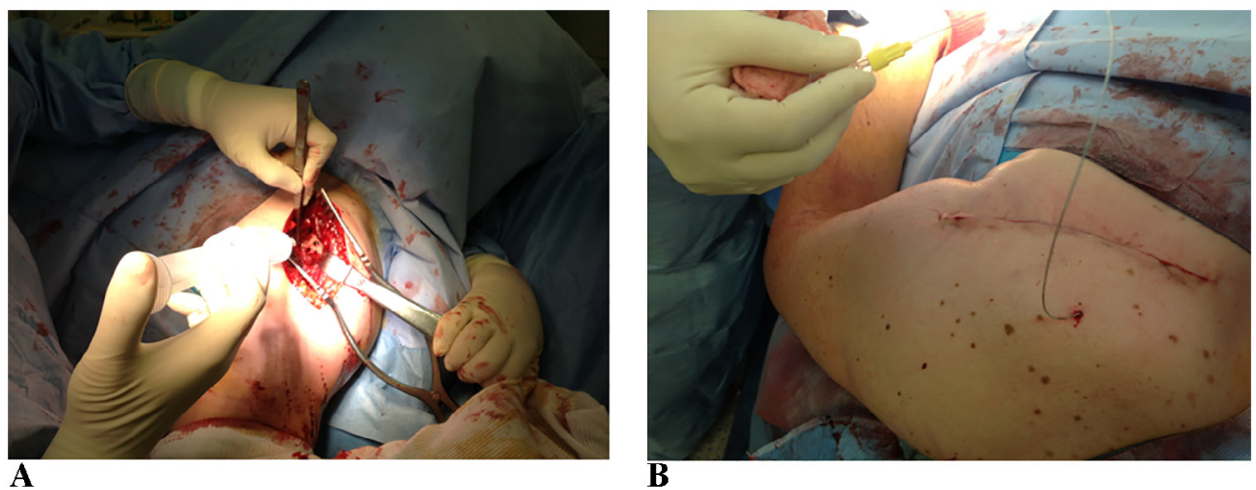

Figure 1 Injection of anesthetic products in situ: infiltration around glenoid (A) and intra-articular catheter (B).

ropivacaine was started at $5 \mathrm{~mL} / \mathrm{h}$ for 48 hours; then, the catheter was removed.

\section{LIA protocol}

LIA was performed by the surgeon. Before placing the TSA components, the surgeon injected $110 \mathrm{~mL}$ of $0.2 \%$ ropivacaine (or $220 \mathrm{mg}$ ) with $30 \mathrm{mg}$ of ketoprofen and $0.5 \mathrm{mg}$ of epinephrine. The infiltration was injected as follows: $50-\mathrm{mL}$ flush in the tissues surrounding the glenoid, 50-mL flush around the humerus into the rotator capsule and cuff, and then $10 \mathrm{~mL}$ in the incision. During closure, the surgeon inserted a catheter connected to an antibacterial filter (20-gauge multiperforated epidural catheter) across the deltoid in the rotator interval (Fig. 1). The next morning, $10 \mathrm{~mL}$ of $0.2 \%$ ropivacaine, $30 \mathrm{mg}$ of ketoprofen, and $0.5 \mathrm{mg}$ of epinephrine were injected through the catheter after clamping the Redon drain (B. Braun, Melsungen, Germany); then, the catheter was removed.

\section{Analgesia protocol}

Whatever the randomization arm, patients systematically received $1 \mathrm{~g}$ of paracetamol associated with tramadol or nefopam perioperatively and postoperatively. If the numerical pain score was above 3 of 10 , a titration of morphine was begun in the recovery room, followed by on-demand oral morphine.

\section{Evaluation criteria}

The primary evaluation criterion was the mean shoulder pain score at rest for the 48-hour postoperative period (on awakening, then at $3,6,12,24,36$, and 48 hours) determined on a numerical scale from 0 (no pain) to 10 (the worst imaginable pain).

The secondary evaluation criteria were mean consumption of opioids (in milligrams of morphine) for the 48-hour postoperative period, the progression of pain at rest and during mobilization (flexion and internal rotation) for the 48-hour postoperative period, the length of the hospital stay, and 1-month postoperative functional recovery of the shoulder according to the Constant score ${ }^{6}$ and the Subjective Shoulder Value (SSV), ${ }^{10}$ which involves asking the patient to rate the operated shoulder compared with a healthy shoulder, which has a score of 100 .

\section{Statistical analyses}

Statistical analyses were performed with STATA software (version 10.0; StataCorp, College Station, TX, USA). The hypotheses to calculate the number of patients were an $\alpha$ risk of 0.025 , a power of 0.90 , and a margin of noninferiority considered to be clinically pertinent of $1 \pm 1.5$ of 10 , with a ratio of $1: 1$. This margin of 1.5 of 10 was slightly superior to the minimum clinically significant change in patient pain severity previously published (1.3 of 10). ${ }^{22}$ The calculated size of the cohort was 94 patients (47 ISB and 47 LIA patients). Once the consent forms were signed, patients were randomized by center on the day of surgery according to a predefined randomization schedule with blocks of 4 . Quantitative variables were tested by the Student $t$ test; qualitative variables, by the $\chi^{2}$ test; and the correlation between continuous variables, by the Pearson $r$ coefficient. $P=.05$ was considered significant.

\section{Results}

\section{Patient characteristics}

A total of 105 patients were eligible for the study. We excluded 6 patients and included 99 patients: 50 in the LIA group and 49 in the ISB group (Fig. 2). The mean age of the patients at inclusion was $72 \pm 9.6$ years, with 35 men and 64 women and 39 left and 60 right shoulders. The 2 groups were comparable at inclusion (Table I).

Of the 99 TSAs, $63(63.6 \%)$ were reverse shoulder arthroplasties whereas $36(36.4 \%)$ were anatomic, with no difference between the ISB and LIA groups $(P=.62)$. No significant difference was found between the 2 groups in the duration of the procedure $(73.3 \pm 26.7$ minutes and $73.4 \pm 17.5$ minutes, respectively; $P=.98)$ or hospital stay ( $4.7 \pm 1.5$ days and $4.8 \pm 1.4$ days, respectively; $P=.72$; range, $1-8$ days).

\section{Primary evaluation criterion}

We found no difference in the mean pain score at rest for the 48-hour postoperative period between the 2 groups, with $1.4 \pm 0.9$ in the LIA group versus $1.7 \pm 1$ in the ISB group $(P=.19)$. 


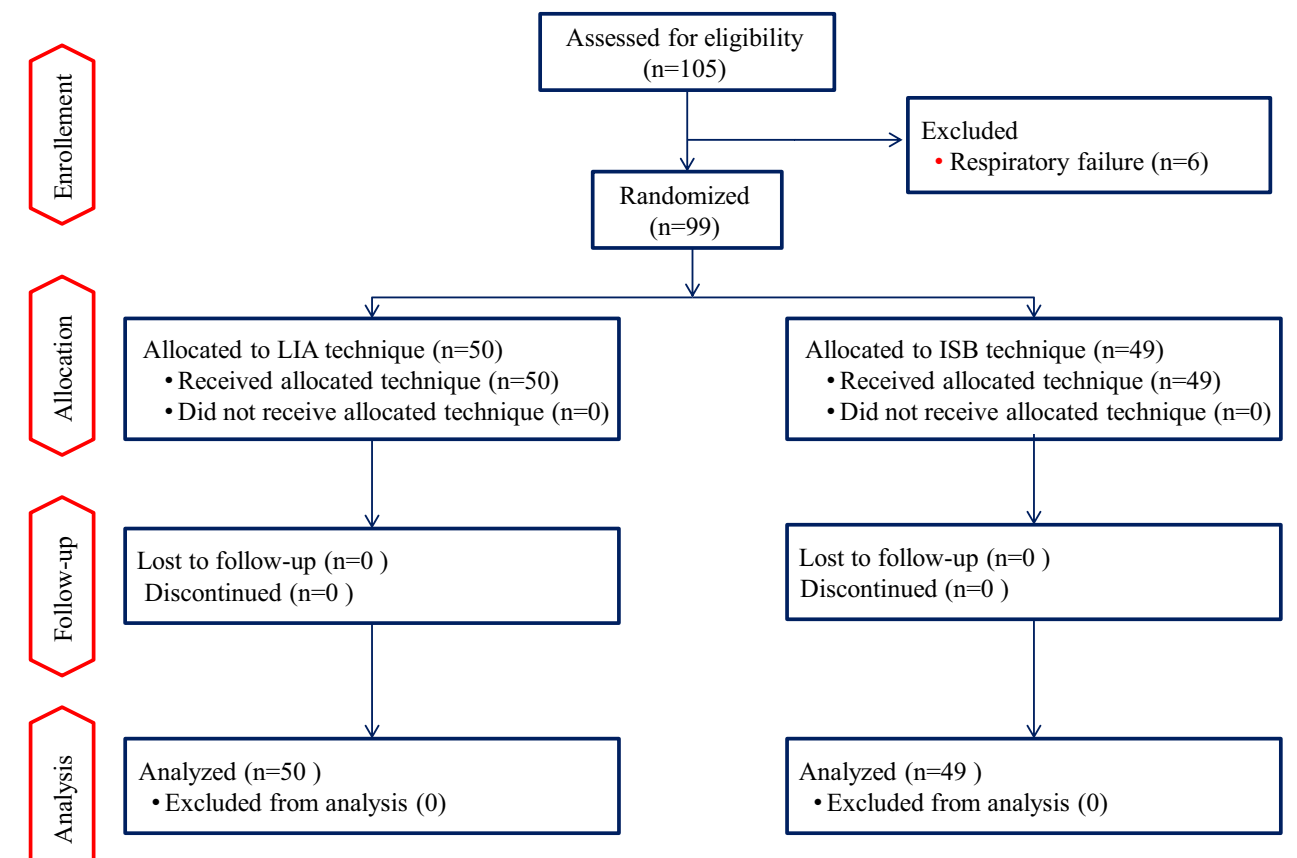

Figure 2 Study flowchart. LIA, local infiltration analgesia; ISB, interscalene block.

Table I Preoperative demographic and clinical patient characteristics

\begin{tabular}{lccc}
\hline & \multicolumn{2}{c}{ Data at inclusion } & P value \\
\cline { 2 - 3 } & LIA $(\mathrm{n}=50)$ & ISB $(\mathrm{n}=49)$ & \\
\hline Age, yr & $\begin{array}{c}72.2 \pm 10.1 \\
(50-91)\end{array}$ & $\begin{array}{c}71.7 \pm 9 \\
(49-89)\end{array}$ & .77 \\
Sex & & & .12 \\
$\quad$ Female & $36(72 \%)$ & $28(57.1 \%)$ & \\
$\quad$ Male & $14(28 \%)$ & $21(42.9 \%)$ & \\
BMI, kg/m & $27 \pm 4.7$ & $27.1 \pm 4.7$ & .92 \\
ASA score & & & .77 \\
1 & $4(8 \%)$ & $5(10.2 \%)$ & \\
2 & $35(70 \%)$ & $31(63.3 \%)$ & \\
3 & $11(22 \%)$ & $13(26.5 \%)$ & \\
Dominant shoulder & $34(68 \%)$ & $32(65.3 \%)$ & .78 \\
Severity of pain (of 10) & $5.6 \pm 2$ & $5.5 \pm 1.9$ & .82 \\
SSV (of 100) & $21.9 \pm 14.4$ & $24.3 \pm 12.9$ & .39 \\
Constant score (of 100) & $33.6 \pm 12.4$ & $37.3 \pm 14.6$ & .18 \\
\hline LIA, local infiltration analgesia; ISB, interscalene nerve block; $B M I$, body \\
mass index; ASA, American Society of Anesthesiologists; SSV, Subjec- \\
tive Shoulder Value.
\end{tabular}

\section{Secondary evaluation criteria}

The progression of pain control at rest was not different between the 2 groups except in the recovery room, where the patients in the LIA group had significantly less pain (Table II, Fig. 3). The patients in the LIA group required less morphine in the recovery room than those in the ISB group $(0.9 \pm 2.4 \mathrm{mg}$ vs $2.4 \pm 3.5 \mathrm{mg}, P=.01)$ (Table III, Fig. 4). On the other hand, no difference in mean 72-hour postoper-
Table II Progression of pain at rest during first 72 hours

\begin{tabular}{llll}
\hline & \multicolumn{2}{l}{$\begin{array}{l}\text { Pain at rest, } \\
\text { mean } \pm \text { SD (of 10) }\end{array}$} & $P$ value \\
\cline { 2 - 3 } & LIA $(\mathrm{n}=50)$ & ISB $(\mathrm{n}=49)$ & \\
\hline In recovery room & $0.6 \pm 1$ & $1.5 \pm 1.7$ & .003 \\
$3 \mathrm{~h}$ & $1.0 \pm 1.5$ & $1.4 \pm 1.6$ & .16 \\
$6 \mathrm{~h}$ & $1.1 \pm 1.3$ & $1.1 \pm 1.2$ & .94 \\
$12 \mathrm{~h}$ & $1.4 \pm 1.4$ & $1.5 \pm 1.5$ & .92 \\
$24 \mathrm{~h}$ & $2.4 \pm 1.9$ & $2.2 \pm 1.9$ & .73 \\
$36 \mathrm{~h}$ & $1.7 \pm 1.5$ & $2.1 \pm 1.6$ & .22 \\
$48 \mathrm{~h}$ & $1.6 \pm 1.5$ & $1.8 \pm 1.4$ & .48 \\
$72 \mathrm{~h}$ & $1.4 \pm 1.4$ & $1.5 \pm 1.2$ & .64 \\
\hline
\end{tabular}

$S D$, standard deviation; $L I A$, local infiltration analgesia; $I S B$, interscalene nerve block.

ative morphine consumption was noted between the 2 groups $(8.4 \pm 11.2 \mathrm{mg}$ in LIA group vs $11.4 \pm 14.1 \mathrm{mg}$ in ISB group, $P=.27)$. We found a strong and significant correlation between the severity of pain and total morphine consumption $(r=0.55$, $P<10^{-5}$ ).

The patients in the LIA group had significantly less severe pain during mobilization on day 2 than those in the ISB group (2.6 \pm 1.4 vs $3.2 \pm 1.3, P=.03)$, but the mean difference was less than the usual minimum clinically significant change in patient pain severity previously published. We found no significant difference in pain during mobilization on day 1 $(2.5 \pm 1.7$ in LIA group vs $3 \pm 1.6$ in ISB group, $P=.19)$ or day $3(2.3 \pm 1.1$ in LIA group vs $2.5 \pm 1.4$ in ISB group, $P=.36$ ) (Fig. 5). 


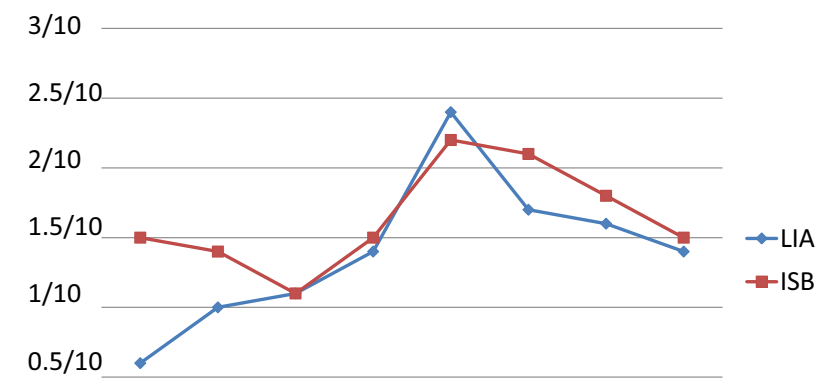

$0 / 10$

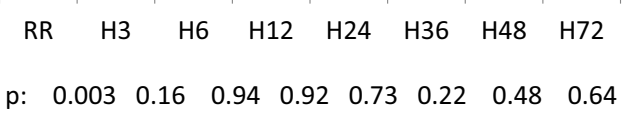

Figure 3 Progression of pain at rest (from 0 [no pain] to 10 [worst imaginable pain]) during first 72 hours $(H)$. LIA, local infiltration analgesia; $I S B$, interscalene block; $R R$, recovery room.

\begin{tabular}{llll} 
Table III & Opioid consumption (noncumulative dose) \\
\hline & \multicolumn{3}{l}{$\begin{array}{l}\text { Noncumulative dose, } \\
\text { mean } \pm \text { SD, mg }\end{array}$} \\
\cline { 2 - 3 } & LIA $(\mathrm{n}=50)$ & ISB $(\mathrm{n}=49)$ & \\
\hline Recovery room & $0.9 \pm 2.4$ & $2.4 \pm 3.5$ & .01 \\
$3 \mathrm{~h}$ & $0.6 \pm 2.1$ & $0.8 \pm 2.1$ & .61 \\
$6 \mathrm{~h}$ & $0.5 \pm 1.8$ & $0.4 \pm 1.7$ & .79 \\
$12 \mathrm{~h}$ & $0.9 \pm 1.2$ & $1.1 \pm 2.9$ & .66 \\
$24 \mathrm{~h}$ & $2.8 \pm 5$ & $2.7 \pm 4.3$ & .92 \\
$36 \mathrm{~h}$ & $1.3 \pm 3$ & $2.1 \pm 3.7$ & .21 \\
$48 \mathrm{~h}$ & $0.8 \pm 2.3$ & $1.1 \pm 3.1$ & .56 \\
$72 \mathrm{~h}$ & $0.5 \pm 1.8$ & $0.7 \pm 3.2$ & .68 \\
\hline
\end{tabular}

SD, standard deviation; LIA, local infiltration analgesia; ISB, interscalene nerve block.

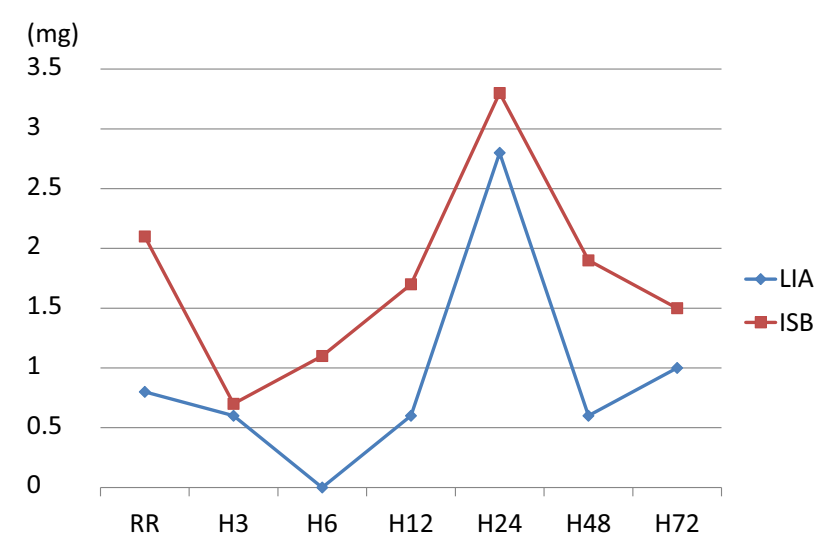

Figure 4 Progression of opioid consumption (in milligrams) during first 72 hours $(H)$. LIA, local infiltration analgesia; ISB, interscalene block; $R R$, recovery room.

A significant improvement in shoulder function scores was noted between preoperative and 1-month postoperative scores, whatever the type of analgesia used, in addition to a decrease in the severity of pain (Table IV). The Constant score

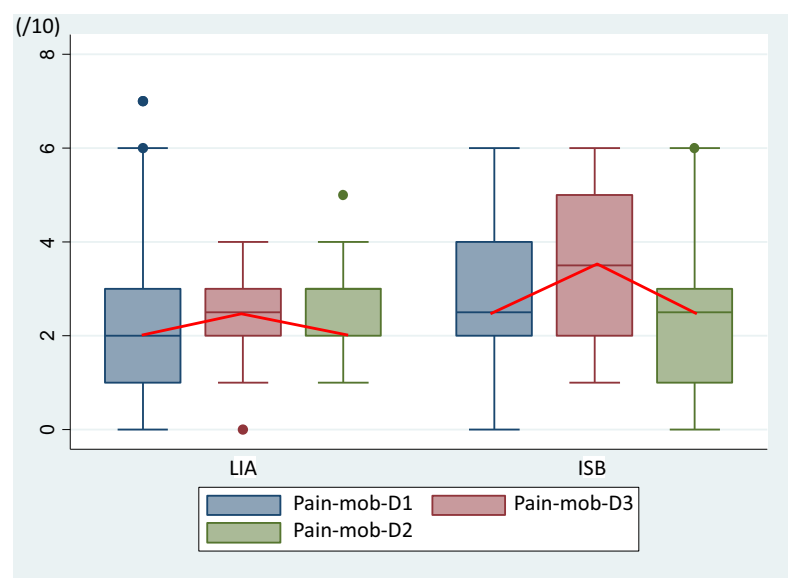

Figure 5 Progression of median pain (from 0 [no pain] to 10 [worst imaginable pain]) during shoulder mobilization $(\mathrm{mob})$ during first 3 postoperative days $(D)$. LIA, local infiltration analgesia; ISB, interscalene block.

Table IV Clinical progression between preoperative data and 1-month follow-up

\begin{tabular}{|c|c|c|c|}
\hline & Preoperatively & $\begin{array}{l}\text { 1-mo } \\
\text { follow-up }\end{array}$ & $P$ value \\
\hline \multicolumn{4}{|l|}{ LIA } \\
\hline $\begin{array}{l}\text { Severity of pain } \\
\quad \text { (of } 10 \text { ) }\end{array}$ & $5.3 \pm 2$ & $1.2 \pm 1.3$ & $<10^{-5}$ \\
\hline SSV (of 100) & $29.3 \pm 17.6$ & $61.2 \pm 12.4$ & $<10^{-5}$ \\
\hline $\begin{array}{l}\text { Constant score } \\
\text { (of 100) }\end{array}$ & $35.8 \pm 13$ & $61.9 \pm 13$ & $<10^{-5}$ \\
\hline \multicolumn{4}{|l|}{ ISB } \\
\hline $\begin{array}{l}\text { Severity of } \\
\quad \text { pain (of 10) }\end{array}$ & $5.3 \pm 2$ & $1.4 \pm 1.6$ & $<10^{-5}$ \\
\hline SSV (of 100) & $27 \pm 14.2$ & $53.54 \pm 14.5$ & $<10^{-5}$ \\
\hline $\begin{array}{l}\text { Constant score } \\
\text { (of 100) }\end{array}$ & $34.9 \pm 15.2$ & $55 \pm 12.2$ & $<10^{-5}$ \\
\hline
\end{tabular}

LIA, local infiltration analgesia; SSV, Subjective Shoulder Value; ISB, interscalene nerve block.

was significantly higher in the LIA group than in the ISB group $(61.9 \pm 13$ vs $54.5 \pm 12.3, P=.04)$, with a difference of $7.4 \%$ (Table IV). The SSV and the severity of shoulder pain were similar in both groups after 1 month: $61.2 \pm 12.4$ in the LIA group versus $53.5 \pm 14.5$ in the ISB group $(P=.05)$ and $1.2 \pm 1.3$ in the LIA group versus $1.4 \pm 1.6$ in the ISB group $(P=.57)$, respectively.

A nonsignificant negative correlation was found between mean pain and function scores for the 48-hour postoperative period after 1 month of follow-up and the Constant score $(r=-0.20, P=.15)$ (Fig. 6) but not the SSV $(r=-0.02$, $P=.88)$.

No severe early postoperative complications associated with the 2 techniques occurred. No catheter-related infections or adverse drug events were reported. Catheter migration occurred in 5 patients $(10.2 \%)$ in the ISB group before the end 


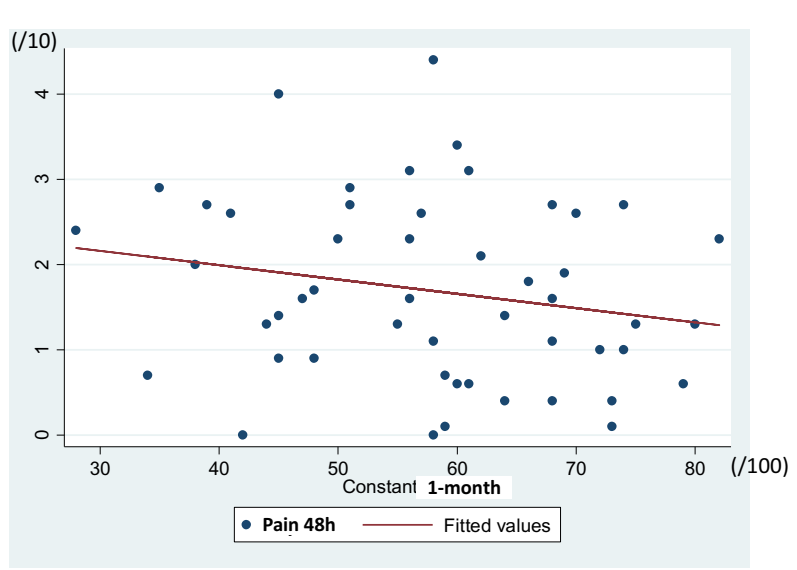

Figure 6 Negative but nonsignificant correlation between mean pain at rest for 48-hour (48h) postoperative period from 0 (no pain) to 10 (worst imaginable pain) and Constant total score (100-point scale) at 1 month of follow-up.

of the 48 hours and $2(4 \%)$ in the LIA group $(P=.22)$. No significant difference in the incidence of nausea and/or vomiting was found between the groups (10\% in LIA group vs $12.2 \%$ in ISB group, $P=.72$ ). On the other hand, $100 \%$ of the patients in the ISB group presented with paresthesia associated with motor block of the operated limb. No revision surgery was required in the first postoperative month.

Several risk factors for early postoperative pain were tested in our study: age $(P=.54)$, sex $(P=.26)$, American Society of Anesthesiologists score $(P=.39)$, surgery on the dominant shoulder $(P=.41)$, and type of implant (anatomic or reverse arthroplasty $(P=.21)$. Only the 7 patients with catheter migration had significantly more pain in the first 48 hours: $3 \pm 0.4$ versus $1.4 \pm 0.8\left(P<10^{-5}\right)$.

\section{Discussion}

This randomized, comparative noninferiority study showed that LIA was not less effective than ISB in controlling shoulder pain for the first 48 hours after TSA. The patients in the LIA group had significantly less pain in the recovery room and consumed significantly less morphine than the patients in the ISB group. A negative but nonsignificant correlation was found between mean pain for the 48-hour postoperative period and the Constant score at 1 month of follow-up.

Other studies have compared the efficacy of LIA and ISB for the same indication. No randomized comparative study has found that ISB is more effective than LIA, whatever the injection protocol or anesthetic used (Table V). The metaanalysis by Wang and Zhang ${ }^{24}$ combined the results of the 4 randomized comparative studies in the literature. ${ }^{2,16,17,25}$ No significant difference in the severity of pain or the consumption of opioids was noted at 12, 24, or 48 hours after surgery.

Other authors have compared protocols combining LIA and ISB. Boddu et $\mathrm{al}^{4}$ evaluated the efficacy of a multimodal analgesic protocol associating ISB $(0.25 \%$ bupivacaine and $4 \mathrm{mg}$ of dexamethasone) and LIA (20 $\mathrm{mL}$ of bupivacaine) in 60 patients who underwent TSA. Postoperative opioids were not required in $64 \%$ of the patients. Sabesan et $\mathrm{al}^{18} \mathrm{com}-$ pared the combination of ISB $(20 \mathrm{~mL}$ of $0.5 \%$ bupivacaine, then $0.125 \%$ bupivacaine at a rate of $6 \mathrm{~mL} / \mathrm{h})$ and LIA $(20 \mathrm{~mL}$ or $266 \mathrm{mg}$ of bupivacaine) versus ISB alone and did not find any significant difference in pain control or opioid consumption in the first 24 hours after surgery.

The volumes and doses of ropivacaine used in our study were high but were not above the threshold of systemic toxicity. This threshold was determined in healthy volunteers,

Table V LIA and ISB protocols for postoperative pain control in patients who underwent shoulder arthroplasty

\begin{tabular}{|c|c|c|c|c|c|}
\hline Authors & Year & Design & Patients & LIA protocol & ISB protocol \\
\hline Weller et $\mathrm{al}^{25}$ & 2017 & RCT & $58 \mathrm{LIA}$ and 156 ISB & $\begin{array}{l}20 \mathrm{~mL} \text { (266 mg) of } 0.5 \% \text { liposomal } \\
\text { bupivacaine, } 10 \mathrm{~mL} \text { of } 0.5 \% \\
\text { bupivacaine with epinephrine, } 2 \mathrm{mg} \text { of } \\
\text { morphine, } 30 \mathrm{mg} \text { of ketorolac } \\
\text { 0.5-mL aliquots via multiple needles }\end{array}$ & $\begin{array}{l}20 \mathrm{~mL} \text { of } 0.5 \% \text { bupivacaine with } \\
1: 200,000 \text { epinephrine } \\
\text { Indwelling ISB }\end{array}$ \\
\hline Namdari et $\mathrm{al}^{16}$ & 2017 & RCT & 78 LIA and 78 ISB & $\begin{array}{l}20 \mathrm{~mL}(266 \mathrm{mg}) \text { of } 1.3 \% \text { bupivacaine } \\
0.5-\mathrm{mL} \text { aliquots via multiple needles }\end{array}$ & $\begin{array}{l}30 \mathrm{~mL} \text { of } 0.5 \% \text { ropivacaine } \\
\text { Single injection under } \\
\text { ultrasound guidance }\end{array}$ \\
\hline Okoroha et $\mathrm{al}^{17}$ & 2016 & RCT & 26 LIA and 31 ISB & $\begin{array}{l}20 \mathrm{~mL}(266 \mathrm{mg} \text { ) of liposomal bupivacaine } \\
1 \text { local injection }\end{array}$ & $\begin{array}{l}40 \mathrm{~mL} \text { of } 0.5 \% \text { ropivacaine } \\
\text { Single injection under } \\
\text { ultrasound guidance }\end{array}$ \\
\hline Abildgaard et $\mathrm{al}^{2}$ & 2017 & $\mathrm{RCT}$ & 37 LIA and 46 ISB & $20 \mathrm{~mL}$ (266 mg) of liposomal bupivacaine & $\begin{array}{l}0.5 \% \text { ropivacaine, } 8 \mathrm{~mL} / \mathrm{h} \\
\text { Indwelling catheter }\end{array}$ \\
\hline Current study & 2018 & $\mathrm{RCT}$ & 50 LIA and 49 ISB & $\begin{array}{l}110 \mathrm{~mL} \text { of } 0.2 \% \\
\text { ropivacaine }(220 \mathrm{mg}), 30 \mathrm{mg} \text { of } \\
\text { ketoprofen, } 0.5 \mathrm{mg} \text { of epinephrine } \\
\text { Day } 1: 10 \mathrm{~mL} \text { of } 0.2 \% \text { ropivacaine, } 30 \mathrm{mg} \\
\text { of ketoprofen, } 0.5 \mathrm{mg} \text { of epinephrine }\end{array}$ & $\begin{array}{l}20 \mathrm{~mL} \text { of } 0.2 \% \text { ropivacaine } \\
0.2 \% \text { ropivacaine, } 5 \mathrm{~mL} / \mathrm{h} \text {, } \\
\text { for } 48 \mathrm{~h}\end{array}$ \\
\hline
\end{tabular}

$L I A$, local infiltration analgesia; ISB, interscalene nerve block; $R C T$, randomized controlled trial. 
and central nervous system adverse effects were found to occur at plasma concentrations greater than $0.6 \mathrm{mg} / \mathrm{L} .{ }^{12}$ Axelsson et $\mathrm{al}^{3}$ measured local anesthetic plasma concentrations after infiltration of $250 \mathrm{mg}$ of ropivacaine for shoulder arthroplasty. The maximum mean concentration was $0.12 \mathrm{mg} / \mathrm{L}$ and was obtained a mean of 2 hours after the bolus injection. The plasma peak concentration during continuous administration of ropivacaine is obtained 24 hours after administration is started. Good clinical tolerance could be linked to slow absorption of ropivacaine, for a mean of 24 hours. Moreover, the addition of a vasoconstrictor such as epinephrine prolongs its effect and reduces vascular absorption. The antiinflammatory ketoprofen was also added to ropivacaine in situ. According to Spreng et al, ${ }^{19}$ local administration of an antiinflammatory is more effective than intravenous administration to control pain.

Certain authors have evaluated the risk of secondary infection due to catheter placement in the surgical site. No reports of early postoperative infection were noted in our study. In a large meta-analysis including more than 2000 patients with an intra-articular catheter, Liu et $\mathrm{al}^{14}$ reported a rate of infection of $0.7 \%$ with a catheter, whatever the location, compared with $1.2 \%$ without a catheter.

Although locoregional anesthesia effectively controls pain, it is time-consuming and limited by its cost and high failure rate, which is approximately $6 \%$ for ISB during shoulder surgery. ${ }^{21}$ Catheter migration is totally unpredictable and may occur in $25 \%-35 \%$ of cases. ${ }^{23}$ Good results depend on correct placement of the catheter near the nerves. In our study, $10.2 \%$ of the perineural catheters migrated during the 48-hour postoperative period in the ISB group compared with $4 \%$ in the LIA group, and mean pain was significantly more severe in the former patients. There is no existing consensus on the type of catheter, the mode of administration, the quantity, or the products to use during LIA. A multiperforated, epiduraltype catheter was chosen because it provides a homogeneous spread over a greater area. The position of the catheter was defined empirically to cover the largest possible portion of the joint. The easy placement of an infiltration catheter is a major advantage compared with perineural catheters. There is no need for anatomic landmarks, and it can be placed under visual control and under optimal aseptic conditions by the surgeon. The technique is therefore much faster to learn, and the failure rate is very low, approximately $1 \% .{ }^{14}$ The absence of a motor block results in faster, more active mobilization of the shoulder, requiring no specific monitoring.

This study has several limitations. The composition of the mixture used in the LIA group and the administration protocol were chosen by the study investigators based on a bibliographic search and several meetings to standardize the protocol. Future comparative studies will determine the optimal LIA protocol for shoulder arthroplasty. Several surgeons and anesthesiologists have participated in this study, but all were experienced and used the described techniques in their daily practice. There was 1 month of follow-up, which is short but similar to the follow-up periods in other studies performed on the subject, which were between 2 months ${ }^{16}$ and 4 months. ${ }^{17}$

\section{Conclusion}

This study shows that LIA is not less effective than ISB for early postoperative pain control following TSA. LIA seems to be a reliable alternative to ISB, in particular in the presence of contraindications to locoregional anesthesia.

\section{Disclaimer}

Philippe Hardy is a consultant for Zimmer. All the other authors, their immediate families, and any research foundations with which they are affiliated have not received any financial payments or other benefits from any commercial entity related to the subject of this article.

\section{References}

1. Abdallah FW, Halpern SH, Aoyama K, Brull R. Will the real benefits of single-shot interscalene block please stand up? A systematic review and meta-analysis. Anesth Analg 2015;120:1114-29. http://dx.doi.org/ 10.1213/ANE.0000000000000688

2. Abildgaard JT, Lonergan KT, Tolan SJ, Kissenberth MJ, Hawkins RJ, Washburn R 3rd, et al. Liposomal bupivacaine versus indwelling interscalene nerve block for postoperative pain control in shoulder arthroplasty: a prospective randomized controlled trial. J Shoulder Elbow Surg 2017;26:1175-81. http://dx.doi.org/10.1016/j.jse.2017.03.012

3. Axelsson K, Nordenson U, Johanzon E, Rawal N, Ekbäck G, Lidegran $\mathrm{G}$, et al. Patient-controlled regional analgesia (PCRA) with ropivacaine after arthroscopic subacromial decompression. Acta Anaesthesiol Scand 2003;47:993-1000. http://dx.doi.org/10.1034/j.1399-6576.2003.00146.x

4. Boddu C, Genza A, McCann PD. Bridging multimodal pain management provides 48-hour pain control in patients undergoing total shoulder replacement. J Shoulder Elbow Surg 2018;27:S65-9. http://dx.doi.org/ 10.1016/j.jse.2017.12.026

5. Busch CA, Whitehouse MR, Shore BJ, MacDonald SJ, McCalden RW, Bourne RB. The efficacy of periarticular multimodal drug infiltration in total hip arthroplasty. Clin Orthop Relat Res 2010;468:2152-9. http://dx.doi.org/10.1007/s11999-009-1198-7

6. Constant CR, Murley AH. A clinical method of functional assessment of the shoulder. Clin Orthop Relat Res 1987;214:160-4.

7. Essving P, Axelsson K, Kjellberg J, Wallgren Ö, Gupta A, Lundin A. Reduced morphine consumption and pain intensity with local infiltration analgesia (LIA) following total knee arthroplasty: a randomized double-blind study involving 48 patients. Acta Orthop 2010;81:354-60. http://dx.doi.org/10.3109/17453674.2010.487241

8. Fredrickson MJ, Leightley P, Wong A, Chaddock M, Abeysekera A, Frampton C. An analysis of 1505 consecutive patients receiving continuous interscalene analgesia at home: a multicentre prospective safety study. Anaesthesia 2016;71:373-9. http://dx.doi.org/10.1111/ anae. 13385

9. Gallay SH, Lobo JJ, Baker J, Smith K, Patel K. Development of a regional model of care for ambulatory total shoulder arthroplasty: a pilot study. Clin Orthop Relat Res 2008;466:563-72. http://dx.doi.org/ 10.1007/s11999-007-0083-5

10. Gilbart MK, Gerber C. Comparison of the subjective shoulder value and the Constant score. J Shoulder Elbow Surg 2007;16:717-21. http:// dx.doi.org/10.1016/j.jse.2007.02.123 
11. Ilfeld BM, Wright TW, Enneking FK, Morey TE. Joint range of motion after total shoulder arthroplasty with and without a continuous interscalene nerve block: a retrospective, case-control study. Reg Anesth Pain Med 2005;30:429-33. http://dx.doi.org/10.1016/j.rapm.2005.06.003

12. Knudsen K, Beckman Suurküla M, Blomberg S, Sjövall J, Edvardsson N. Central nervous and cardiovascular effects of i.v. infusions of ropivacaine, bupivacaine and placebo in volunteers. Br J Anaesth 1997;78:507-14.

13. Lenters TR, Davies J, Matsen FA 3rd. The types and severity of complications associated with interscalene brachial plexus block anesthesia: local and national evidence. J Shoulder Elbow Surg 2007;16:379-87. http://dx.doi.org/10.1016/j.jse.2006.10.007

14. Liu SS, Richman JM, Thirlby RC, Wu CL. Efficacy of continuous wound catheters delivering local anesthetic for postoperative analgesia: a quantitative and qualitative systematic review of randomized controlled trials. J Am Coll Surg 2006;203:914-32. http://dx.doi.org/10.1016/ j.jamcollsurg.2006.08.007

15. Mahoney A, Bosco JA 3rd, Zuckerman JD. Readmission after shoulder arthroplasty. J Shoulder Elbow Surg 2014;23:377-81. http://dx.doi.org/ 10.1016/j.jse.2013.08.007

16. Namdari S, Nicholson T, Abboud J, Lazarus M, Steinberg D, Williams G. Randomized controlled trial of interscalene block compared with injectable liposomal bupivacaine in shoulder arthroplasty. J Bone Joint Surg Am 2017;99:550-6. http://dx.doi.org/10.2106/JBJS.16.00296

17. Okoroha KR, Lynch JR, Keller RA, Korona J, Amato C, Rill B, et al. Liposomal bupivacaine versus interscalene nerve block for pain control after shoulder arthroplasty: a prospective randomized trial. J Shoulder Elbow Surg 2016;25:1742-8. http://dx.doi.org/10.1016/j.jse.2016.05.007

18. Sabesan VJ, Shahriar R, Petersen-Fitts GR, Whaley JD, Bou-Akl T, Sweet M, et al. A prospective randomized controlled trial to identify the optimal postoperative pain management in shoulder arthroplasty: liposomal bupivacaine versus continuous interscalene catheter. J Shoulder Elbow Surg 2017;26:1810-7. http://dx.doi.org/ 10.1016/j.jse.2017.06.044

19. Spreng UJ, Dahl V, Hjall A, Fagerland MW, Raeder J. High-volume local infiltration analgesia combined with intravenous or local ketorolac+morphine compared with epidural analgesia after total knee arthroplasty. Br J Anaesth 2010;105:675-82. http://dx.doi.org/10.1093/ $\mathrm{bja} / \mathrm{aeq} 232$

20. Sviggum HP, Jacob AK, Mantilla CB, Schroeder DR, Sperling JW, Hebl JR. Perioperative nerve injury after total shoulder arthroplasty: assessment of risk after regional anesthesia. Reg Anesth Pain Med 2012;37:490-4. http://dx.doi.org/10.1097/AAP.0b013e31825c258b

21. Tetzlaff JE, Yoon HJ, Brems J. Interscalene brachial plexus block for shoulder surgery. Reg Anesth 1994;19:339-43.

22. Todd K, Funk K, Funk J, Bonacci R. Clinical significance of reported changes in pain severity. Ann Emerg Med 1996;27:485-9.

23. Tuominen M, Haasio J, Hekali R, Rosenberg PH. Continuous interscalene brachial plexus block: clinical efficacy, technical problems and bupivacaine plasma concentrations. Acta Anaesthesiol Scand 1989;33:848.

24. Wang K, Zhang HX. Liposomal bupivacaine versus interscalene nerve block for pain control after total shoulder arthroplasty: a systematic review and meta-analysis. Int J Surg 2017;46:61-70. http://dx.doi.org/ 10.1016/j.ijsu.2017.08.569

25. Weller WJ, Azzam MG, Smith RA, Azar FM, Throckmorton TW. Liposomal bupivacaine mixture has similar pain relief and significantly fewer complications at less cost compared to indwelling interscalene catheter in total shoulder arthroplasty. J Arthroplasty 2017;32:3557-62. http://dx.doi.org/10.1016/j.arth.2017.03.017 